\title{
Brugada phenocopy unmasked during a febrile illness
}

\author{
Devasmitha Wijesundara ${ }^{1}$, Keshinie Samarasekara $^{1}$, Thashi Chang ${ }^{1,2}$
}

Ceylon Medical Journal 2020; 65: 120-121

DOI: http://doi.org/10.4038/cmj.v65i4.9284

\section{Introduction}

A 40-year-old previously healthy man was treated for dengue haemorrhagic fever (DHF), with a positive NS1 antigen test on day 2 of fever and sonographic evidence of fluid leakage into the hepatorenal pouch on day 6 . A routine ECG done during the peak of the febrile phase of the DHF showed "coved” ST segment elevations in lead V1 which is characteristic of type 1 Brugada syndrome (Figure 1 and Figure 2A). However, the patient did not have any cardiac symptoms, nor did he report any personal or family history of premature cardiac events. His echocardiogram, serum troponin I and electrolytes were normal. The ST segment elevation on ECG completely resolved parallel to the resolution of fever (Figure 1 and Figures 2B and 2C).

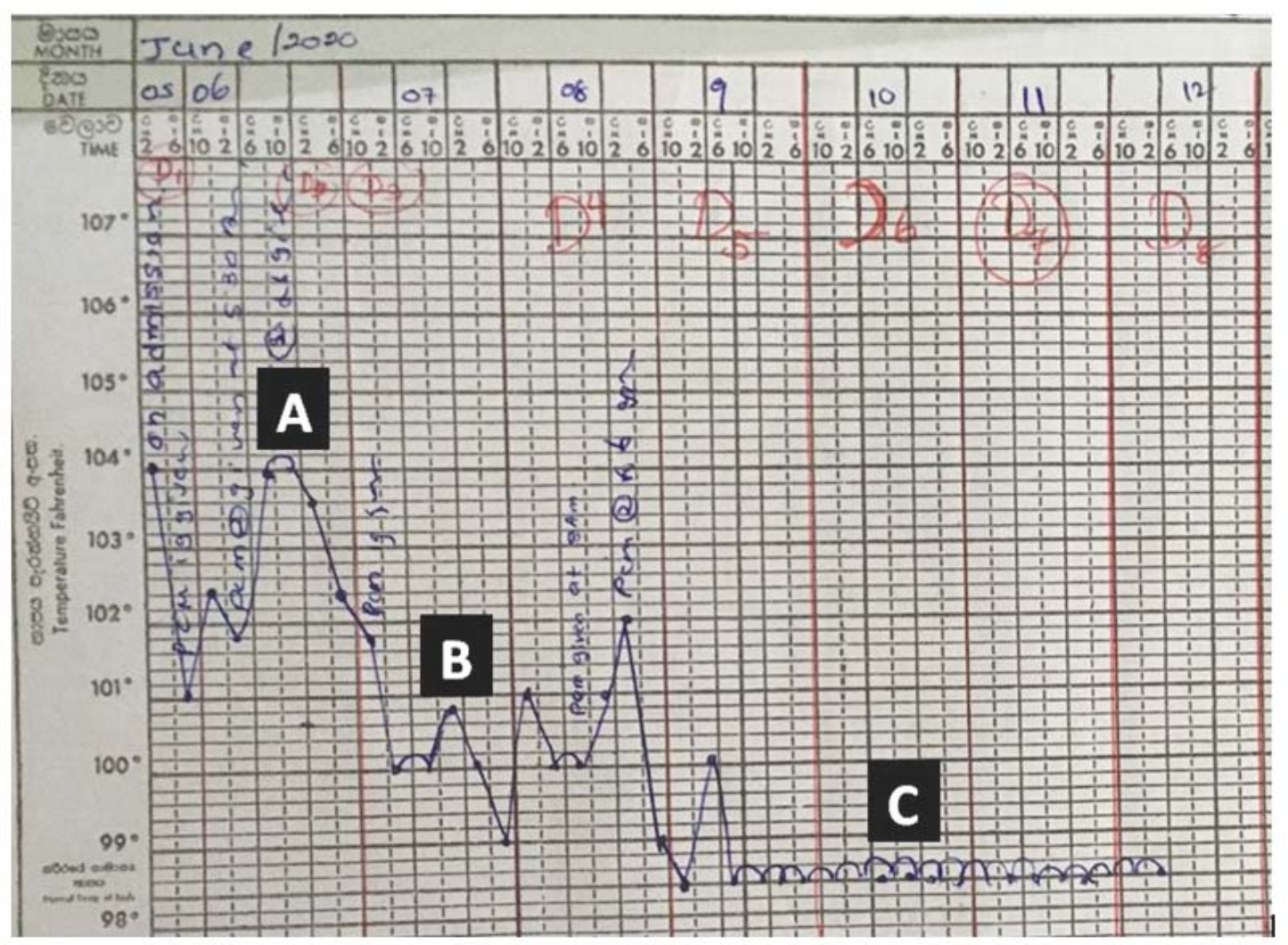

Figure 1. Patient's temperature chart; A - C indicate the time points at which ECG was recorded.

${ }^{1}$ Professorial Unit in Medicine, National Hospital of Sri Lanka, Colombo. ${ }^{2}$ Department of Clinical Medicine, Faculty of Medicine, University of Colombo, Sri Lanka.

Correspondence: DW, e-mail: <devasaw@gmail.com>. Received 07 October 2020 and revised version 08 December 2020 accepted 02 December 2020.

This is an open-access article distributed under the terms of the Creative Commons Attribution License, which permits unrestricted use, distribution, and reproduction in any medium, provided the original author and source are credited. 


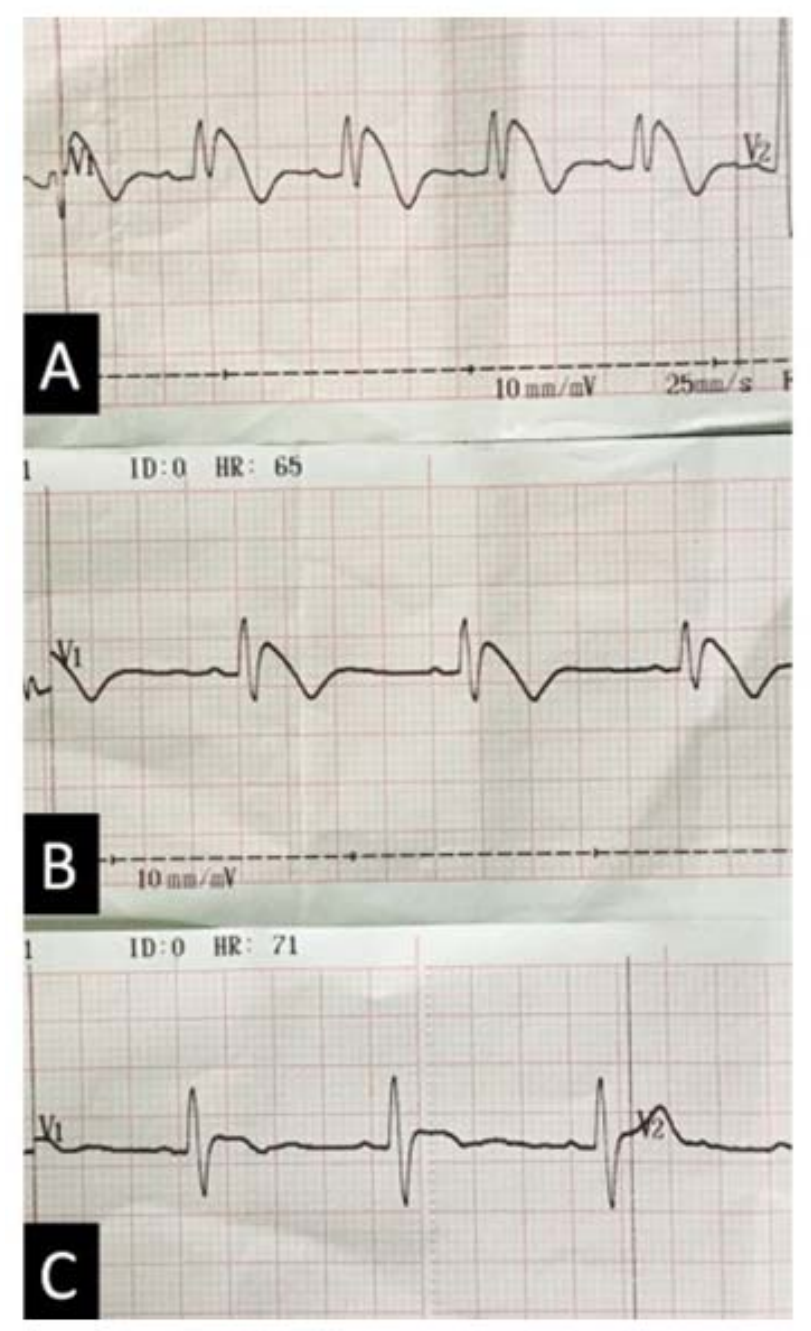

Figure 2. Serial ECG recordings demonstrating resolution of the Brugada pattern in lead V1.

Brugada syndrome (BrS) is an autosomal dominant cardiac channelopathy associated with an increased risk of malignant arrhythmias leading to syncope and cardiac arrest. Loss of function SCN5A (sodium channel) mutations are present in around 20\% of patients [1]. Fever is known to unmask Brugada syndrome.

Incidental discovery of a Brugada pattern on ECG in the absence of the syndrome is known as Brugada phenocopy (BrP) [2]. Complete resolution of ECG changes after reversal of the underlying condition characterizes BrP. Provocative testing with sodium channel blockers is negative in $\mathrm{BrP}$ in contrast to $\mathrm{BrS}$, but was not carried out in this patient due to the risks associated with the test and because the absence of past, present or family histories of cardiac events favoured BrP. This patient fulfilled diagnostic criteria for BrP - Class B (highly suspicious BrP) [3].

\section{Consent for publication}

Informed written consent was obtained from the patient for publication of this case report and any accompanying images.

\section{References}

1. Grant AO. Electrophysiological Basis and Genetics of Brugada Syndrome. J Cardiovasc Electrophysiol. 2005; 16(9): 3-7.

2. Anselm DD, Evans JM, Baranchuk A. Brugada phenocopy: A new electrocardiogram phenomenon. World J Cardiol. 2014; 6(3): 81-6.

3. Gottschalk B, Anselm DD, Baranchuk A. Brugada phenocopy: morphological classification and importance of provocative testing. Ann Noninvasive Electrocardiol. 2014; 19(6): 604-5. 